\title{
Existence of Weak Solutions for Second-Order Boundary Value Problem of Impulsive Dynamic Equations on Time Scales
}

\author{
Hongbo Duan and Hui Fang \\ Department of Applied Mathematics, Kunming University of Science and Technology, \\ Kunming, Yunnan 650093, China \\ Correspondence should be addressed to Hui Fang, kmustfanghui@hotmail.com \\ Received 9 April 2009; Accepted 28 June 2009 \\ Recommended by Victoria Otero-Espinar
}

We study the existence of weak solutions for second-order boundary value problem of impulsive dynamic equations on time scales by employing critical point theory.

Copyright (c) 2009 H. Duan and H. Fang. This is an open access article distributed under the Creative Commons Attribution License, which permits unrestricted use, distribution, and reproduction in any medium, provided the original work is properly cited.

\section{Introduction}

Consider the following boundary value problem:

$$
\begin{gathered}
-u^{\Delta \Delta}(t)=f\left(\sigma(t), u^{\sigma}(t)\right), \quad t \in[0, T]_{\mathbb{T}}, t \neq t_{j}, j=1,2, \ldots, p, \\
u\left(t_{j}^{+}\right)-u\left(t_{j}^{-}\right)=A_{j} u\left(t_{j}^{-}\right), \quad j=1,2, \ldots, p, \\
u^{\Delta}\left(t_{j}^{+}\right)-u^{\Delta}\left(t_{j}^{-}\right)=B_{j} u^{\Delta}\left(t_{j}^{-}\right)+I_{j}\left(u\left(t_{j}^{-}\right)\right), \quad j=1,2, \ldots, p, \\
u(0)=0=u(T),
\end{gathered}
$$

where $\mathbb{T}$ is a time scale, $[0, T]_{\mathbb{T}}:=[0, T] \cap \mathbb{T}, \sigma(0)=0$ and $\sigma(T)=T, f:[0, T]_{\mathbb{T}} \times \mathbb{R} \rightarrow \mathbb{R}$ is a given function, $I_{j} \in C[\mathbb{R}, \mathbb{R}],\left\{A_{j}\right\},\left\{B_{j}\right\}$ are real sequences with $B_{j}=\left(1+A_{j}\right)^{-1}-1$ and $\sum_{k=1}^{p}\left|A_{k}\right|<1$, the impulsive points $t_{j} \in[0, T]_{\mathbb{T}}$ are right-dense and $0=t_{0}<t_{1}<\cdots<t_{p}<$ $t_{p+1}=T, \lim _{h \rightarrow 0^{+}} u^{\Delta}\left(t_{j}+h\right)$ and $\lim _{h \rightarrow 0^{+}} u^{\Delta}\left(t_{j}-h\right)$ represent the right and left limits of $u^{\Delta}(t)$ at $t=t_{j}$ in the sense of the time scale, that is, in terms of $h>0$ for which $t_{j}+h, t_{j}-h \in[0, T]_{\mathbb{T}}$, whereas if $t_{j}$ is left-scattered, we interpret $u^{\Delta}\left(t_{j}^{-}\right)=u^{\Delta}\left(t_{j}\right)$ and $u\left(t_{j}^{-}\right)=u\left(t_{j}\right)$. 
The theory of time scales, which unifies continuous and discrete analysis, was first introduced by Hilger [1]. The study of boundary value problems for dynamic equations on time scales has recently received a lot of attention, see [2-16]. At the same time, there have been significant developments in impulsive differential equations, see the monographs of Lakshmikantham et al. [17] and Samollenko and Perestyuk [18]. Recently, Benchohra and Ntouyas [19] obtained some existence results for second-order boundary value problem of impulsive differential equations on time scales by using Schaefer's fixed point theorem and nonlinear alternative of Leray-Schauder type. However, to the best of our knowledge, few papers have been published on the existence of solutions for second-order boundary value problem of impulsive dynamic equations on time scales via critical point theory. Inspired and motivated by Jiang and Zhou [10], Nieto and O'Regan [20], and Zhang and Li [21], we study the existence of weak solutions for boundary value problems of impulsive dynamic equations on time scales (1.1)-(1.4) via critical point theory.

This paper is organized as follows. In Section 2, we present some preliminary results concerning the time scales calculus and Sobolev's spaces on time scales. In Section 3, we construct a variational framework for (1.1)-(1.4) and present some basic notation and results. Finally, Section 4 is devoted to the main results and their proof.

\section{Preliminaries about Time Scales}

In this section, we briefly present some fundamental definitions and results from the calculus on time scales and Sobolev's spaces on time scales so that the paper is self-contained. For more details, one can see [22-25].

Definition 2.1. A time scale $\mathbb{T}$ is an arbitrary nonempty closed subset of $\mathbb{R}$, equipped with the topology induced from the standard topology on $\mathbb{R}$.

$$
\text { For } a, b \in \mathbb{T}, a<b,[a, b]_{\mathbb{T}}:=[a, b] \cap \mathbb{T},[a, b)_{\mathbb{T}}:=[a, b) \cap \mathbb{T} .
$$

Definition 2.2. One defines the forward jump operator $\sigma: \mathbb{T} \rightarrow \mathbb{T}$, the backward jump operator $\rho: \mathbb{T} \rightarrow \mathbb{T}$, and the graininess $\mu: \mathbb{T} \rightarrow \mathbb{R}^{+}=[0, \infty)$ by

$$
\sigma(t):=\inf \{s \in \mathbb{T}: s>t\}, \quad \rho(t):=\sup \{s \in \mathbb{T}: s<t\}, \quad \mu(t)=\sigma(t)-t \quad \text { for } t \in \mathbb{T},
$$

respectively. If $\sigma(t)=t$, then $t$ is called right-dense (otherwise: right-scattered), and if $\rho(t)=t$, then $t$ is called left-dense (otherwise: left-scattered). Denote $y^{\sigma}(t)=y(\sigma(t))$.

Definition 2.3. Assume $f: \mathbb{T} \rightarrow \mathbb{R}$ is a function and let $t \in \mathbb{T}$. Then one defines $f^{\Delta}(t)$ to be the number (provided it exists) with the property that given any $\varepsilon>0$, there is a neighborhood $U$ of $t$ (i.e., $U=(t-\delta, t+\delta) \cap \mathbb{T}$ for some $\delta>0$ ) such that

$$
\left|[f(\sigma(t))-f(s)]-f^{\Delta}(t)[\sigma(t)-s]\right| \leq \varepsilon|\sigma(t)-s| \quad \forall s \in U
$$

In this case, $f^{\Delta}(t)$ is called the delta (or Hilger) derivative of $f$ at $t$. Moreover, $f$ is said to be delta or Hilger differentiable on $\mathbb{T}$ if $f^{\Delta}(t)$ exists for all $t \in \mathbb{T}$. 
Definition 2.4. A function $f: \mathbb{T} \rightarrow \mathbb{R}$ is said to be rd-continuous if it is continuous at rightdense points in $\mathbb{T}$ and its left-sided limits exist (finite) at left-dense points in $\mathbb{T}$. The set of rd-continuous functions $f: \mathbb{T} \rightarrow \mathbb{R}$ will be denoted by $C_{\mathrm{rd}}(\mathbb{T})$.

As mentioned in [24], the Lebesgue $\mu_{\Delta}$-measure can be characterized as follows:

$$
\mu_{\Delta}=\lambda+\sum_{i \in I}\left(\sigma\left(t_{i}\right)-t_{i}\right) \delta_{t_{i}}
$$

where $\lambda$ is the Lebesgue measure on $\mathbb{R}$, and $\left\{t_{i}\right\}_{i \in I}$ is the (at most countable) set of all right-scattered points of $\mathbb{T}$. A function $f$ which is measurable with respect to $\mu_{\Delta}$ is called $\Delta$-measurable, and the Lebesgue integral over $[a, b)_{\mathbb{T}}$ is denoted by

$$
\int_{a}^{b} f(t) \Delta t:=\int_{[a, b)_{\mathbb{T}}} f(t) d \mu_{\Delta}
$$

The Lebesgue integral associated with the measure $\mu_{\Delta}$ on $\mathbb{T}$ is called the Lebesgue delta integral.

Lemma 2.5 (see [24, Theorem 2.11]). If $f, g:[a, b]_{\mathbb{T}} \rightarrow \mathbb{R}$ are absolutely continuous functions on $[a, b]_{\mathbb{T}}$, then $f \cdot g$ is absolutely continuous on $[a, b]_{\mathbb{T}}$ and the following equality is valid:

$$
\int_{[a, b)_{\mathbb{T}}} f(t) g^{\Delta}(t) \Delta t=\left.[f(t) g(t)]\right|_{a} ^{b}-\int_{[a, b)_{\mathbb{T}}} f^{\Delta}(t) g^{\sigma}(t) \Delta t
$$

For $1<p<\infty$, the Banach space $L_{\Delta}^{p}$ may be defined in the standard way, namely,

$$
L_{\Delta}^{p}\left([a, b)_{\mathbb{T}}\right):=\left\{f:[a, b]_{\mathbb{T}} \longrightarrow \mathbb{R} \mid f \text { is } \Delta \text {-measurable and } \int_{a}^{b}|f(t)|^{p} \Delta t<\infty\right\},
$$

equipped with the norm

$$
\|f\|_{L_{\Delta}^{p}}:=\left(\int_{a}^{b}|f(t)|^{p} \Delta t\right)^{1 / p}
$$

Let $H_{\Delta}^{1}\left([a, b]_{\mathbb{T}}\right)$ be the space of the form

$$
\begin{aligned}
H_{\Delta}^{1}\left([a, b]_{\mathbb{T}}\right) & :=W_{\Delta}^{1,2}\left([a, b]_{\mathbb{T}}\right) \\
& :=\left\{f:[0, T]_{\mathbb{T}} \longrightarrow \mathbb{R} \mid f \text { is absolutely continuous on }[a, b]_{\mathbb{T}}, f^{\Delta} \in L_{\Delta}^{2}\left([a, b)_{\mathbb{T}}\right)\right\}
\end{aligned}
$$


its norm is induced by the inner product given by

$$
(f, g)_{H_{\Delta}^{1}}:=\int_{a}^{b} f^{\Delta}(t) g^{\Delta}(t) \Delta t+\int_{a}^{b} f(t) g(t) \Delta t
$$

for all $f, g \in H_{\Delta}^{1}\left([a, b]_{\mathbb{T}}\right)$.

Let $C\left([a, b]_{\mathbb{T}}\right)$ denote the linear space of all continuous function $f:[a, b]_{\mathbb{T}} \rightarrow \mathbb{R}$ with the maximum norm $\|f\|_{C}=\max _{t \in[a, b]_{\mathbb{T}}}|f(t)|$.

Lemma 2.6 (see [24, Corollary 3.8]). Let $\left\{x_{m}\right\} \subset H_{\Delta}^{1}\left([a, b]_{\mathbb{T}}\right)$, and $x \in H_{\Delta}^{1}\left([a, b]_{\mathbb{T}}\right)$. If $\left\{x_{m}\right\}$ converges weakly in $H_{\Delta}^{1}\left([a, b]_{\mathbb{T}}\right)$ to $x$, then $\left\{x_{m}\right\}$ converges strongly in $C\left([a, b]_{\mathbb{T}}\right)$ to $x$.

Lemma 2.7 (Hölder inequality [25, Theorem 3.1]). Let $f, g \in C_{\mathrm{rd}}([a, b]), p>1$ and $q$ be the conjugate number of $p$. Then

$$
\int_{a}^{b}|f(t) g(t)| \Delta t \leq\left(\int_{a}^{b}|f(t)|^{p} \Delta t\right)^{1 / p}\left(\int_{a}^{b}|g(t)|^{q} \Delta t\right)^{1 / q} .
$$

When $p=q=2$, we obtain the Cauchy-Schwarz inequality.

For more basic properties of Sobolev's spaces on time scales, one may refer to Agarwal et al. [24].

\section{Variational Framework}

In this section, we will establish the corresponding variational framework for problem (1.1)(1.4).

Let $\Gamma_{j}=\left[t_{j}, t_{j+1}\right]_{\mathbb{T}}$, and

$$
f_{\Gamma_{j}}(t):= \begin{cases}f(t), & t \in\left(t_{j}, t_{j+1}\right]_{\mathbb{T}^{\prime}} \\ f\left(t_{j}^{+}\right), & t=t_{j}\end{cases}
$$

for $j=0,1, \ldots, p$.

Now we consider the following space:

$H:=\left\{f:[0, T]_{\mathbb{T}} \longrightarrow \mathbb{R} \mid f\right.$ is continuous from left at each $t_{j}, f\left(t_{j}^{+}\right)$exists,

$f_{\Gamma_{j}}$ is absolutely continuous on $\Gamma_{j}$, the delta derivative of $f_{\Gamma_{j}} \in L_{\Delta}^{2}\left(\left[t_{j}, t_{j+1}\right)_{\mathbb{T}}\right)$,

$f$ satisfies the condition (1.2) for all $j=0,1, \ldots, p, f(0)=f(T)=0\}$, 
its norm is induced by the inner product given by

$$
(f, g)_{H}:=\sum_{j=0}^{p} \int_{t_{j}}^{t_{j+1}} f_{\Gamma_{j}}^{\Delta}(t) g_{\Gamma_{j}}^{\Delta}(t) \Delta t, \quad \forall f, g \in H .
$$

That is

$$
\|f\|_{H}=\left(\sum_{j=0}^{p} \int_{t_{j}}^{t_{j+1}}\left|f_{\Gamma_{j}}^{\Delta}(t)\right|^{2} \Delta t\right)^{1 / 2}
$$

for any $f \in H$.

First, we give some lemmas which are useful in the proof of theorems.

Lemma 3.1. If $\sum_{k=1}^{p}\left|A_{k}\right|<1$, then for any $x \in H$, $\sup _{t \in[0, T]_{\mathbb{T}}}|x(t)| \leq R_{0}\|x\|_{H}$, where $R_{0}=$ $T^{1 / 2} /\left(1-\sum_{k=1}^{p}\left|A_{k}\right|\right)$.

Proof. For any $x \in H$ and $t \in\left[t_{j}, t_{j+1}\right]_{\mathbb{T}}, j=0,1, \ldots, p$, we have

$$
\begin{aligned}
|x(t)| & =\left|x(t)-x\left(t_{j}^{+}\right)+x\left(t_{j}^{+}\right)-\cdots-x\left(t_{1}^{+}\right)+x\left(t_{1}^{+}\right)-x\left(t_{0}\right)\right| \\
& =\left|x(t)-x\left(t_{j}^{+}\right)+\sum_{k=0}^{j-1}\left[x\left(t_{k+1}^{-}\right)-x\left(t_{k}^{+}\right)\right]+\sum_{k=0}^{j-1} A_{k+1} x\left(t_{k+1}^{-}\right)\right| \\
& =\left|\int_{t_{j}}^{t} x_{\Gamma_{k}}^{\Delta}(s) \Delta s+\sum_{k=0}^{j-1} \int_{t_{k}}^{t_{k+1}} x_{\Gamma_{k}}^{\Delta}(s) \Delta s+\sum_{k=0}^{j-1} A_{k+1} x\left(t_{k+1}^{-}\right)\right| \\
& \leq \int_{t_{j}}^{t}\left|x_{\Gamma_{j}}^{\Delta}(s)\right| \Delta s+\sum_{k=0}^{j-1} \int_{t_{k}}^{t_{k+1}}\left|x_{\Gamma_{k}}^{\Delta}(s)\right| \Delta s+\sum_{k=0}^{j-1}\left|A_{k+1}\right|\left|x\left(t_{k+1}^{-}\right)\right| \\
& \leq \sum_{k=0}^{p} \int_{t_{k}}^{t_{k+1}}\left|x_{\Gamma_{k}}^{\Delta}(s)\right| \Delta s+\sum_{k=0}^{j-1}\left|A_{k+1}\right|\left|x\left(t_{k+1}^{-}\right)\right| \\
& \leq T^{1 / 2}\|x\|_{H}+\sum_{k=1}^{p}\left|A_{k}\right| \sup _{t \in[0, T]_{\mathbb{T}}}|x(t)|,
\end{aligned}
$$

which implies that

$$
\sup _{t \in[0, T]_{\mathbb{T}}}|x(t)| \leq R_{0}\|x\|_{H^{\prime}} \quad \forall x \in H .
$$

Lemma 3.2. $H$ is a Hilbert space. 
Proof. Let $\left\{u_{k}\right\}_{k=1}^{\infty}$ be a Cauchy sequence in H. By Lemma 3.1, we have

$$
\begin{aligned}
\|f\|_{H_{\Delta}^{1}\left(\left[t_{j}, t_{j+1}\right]_{\mathbb{T}}\right)} & =\left[\int_{t_{j}}^{t_{j+1}}\left|f_{\Gamma_{j}}^{\Delta}(t)\right|^{2} \Delta t+\int_{t_{j}}^{t_{j+1}}\left|f_{\Gamma_{j}}(t)\right|^{2} \Delta t\right]^{1 / 2} \\
& \leq\left[\int_{t_{j}}^{t_{j+1}}\left|f_{\Gamma_{j}}^{\Delta}(t)\right|^{2} \Delta t+R_{0}^{2}\left(t_{j+1}-t_{j}\right)\|f\|_{H}^{2}\right]^{1 / 2} \\
& \leq\left(1+R_{0}^{2} T\right)^{1 / 2}\|f\|_{H} .
\end{aligned}
$$

Set

$$
u_{k}^{j}(t):=\left(u_{k}\right)_{\Gamma_{j}}:= \begin{cases}u_{k}(t), & t \in\left(t_{j}, t_{j+1}\right]_{\mathbb{T}^{\prime}} \\ u_{k}\left(t_{j}^{+}\right), & t=t_{j},\end{cases}
$$

for $j=0,1, \ldots, p, k=1,2, \ldots$ Then $\left\{u_{k}^{j}\right\}_{k=1}^{\infty}$ be a Cauchy sequence in $H_{\Delta}^{1}\left(\left[t_{j}, t_{j+1}\right]_{\mathbb{T}}\right)$, for $j=0,1, \ldots, p$. Therefore, there exists a $u^{j} \in H_{\Delta}^{1}\left(\left[t_{j}, t_{j+1}\right]_{\mathbb{T}}\right)$, such that $\left\{u_{k}^{j}\right\}$ converges to $u^{j}$ in $H_{\Delta}^{1}\left(\left[t_{j}, t_{j+1}\right]_{\mathbb{T}}\right), j=0,1, \ldots, p$. It follows from Lemma 2.6 that $\left\{u_{k}^{j}\right\}$ converges strongly to $u^{j}$ in $C\left(\left[t_{j}, t_{j+1}\right]_{\mathbb{T}}\right)$, that is, $\left\|u_{k}^{j}-u^{j}\right\|_{C\left(\left[t_{j}, t_{j+1}\right]_{\mathbb{T}}\right)} \rightarrow 0$ as $k \rightarrow+\infty$ for all $j=0,1, \ldots, p$. Hence, we have

$$
\lim _{k \rightarrow+\infty} u_{k}^{j}\left(t_{j}\right)=u^{j}\left(t_{j}\right), \quad \lim _{k \rightarrow+\infty} u_{k}^{j-1}\left(t_{j}\right)=u^{j-1}\left(t_{j}\right)
$$

Noting that

$$
\begin{aligned}
\lim _{k \rightarrow+\infty} u_{k}^{j}\left(t_{j}\right) & =\lim _{k \rightarrow+\infty} u_{k}\left(t_{j}^{+}\right)=\lim _{k \rightarrow+\infty}\left(1+A_{j}\right) u_{k}\left(t_{j}^{-}\right)=\lim _{k \rightarrow+\infty}\left(1+A_{j}\right) u_{k}\left(t_{j}\right) \\
& =\left(1+A_{j}\right) \lim _{k \rightarrow+\infty} u_{k}^{j-1}\left(t_{j}\right)=\left(1+A_{j}\right) u^{j-1}\left(t_{j}\right),
\end{aligned}
$$

we have

$$
u^{j}\left(t_{j}\right)=\left(1+A_{j}\right) u^{j-1}\left(t_{j}\right), \quad j=0,1, \ldots, p
$$

Set

$$
u(t):= \begin{cases}u^{j}(t), & t \in\left(t_{j}, t_{j+1}\right]_{\mathbb{T}^{\prime}} j=0,1, \ldots, p, \\ u^{j-1}\left(t_{j}\right), & t=t_{j}, j=0,1, \ldots, p .\end{cases}
$$


Then we have

$$
\begin{gathered}
u\left(t_{j}^{+}\right)=u^{j}\left(t_{j}^{+}\right)=u^{j}\left(t_{j}\right)= \\
\left(1+A_{j}\right) u^{j-1}\left(t_{j}\right)=\left(1+A_{j}\right) u\left(t_{j}\right)=\left(1+A_{j}\right) u\left(t_{j}^{-}\right) \\
u_{\Gamma_{j}}=u^{j}, \quad j=0,1, \ldots, p
\end{gathered}
$$

Thus $u \in H$. Noting that

$$
\begin{aligned}
\left\|u_{k}-u\right\|_{H} & =\left[\sum_{j=0}^{p} \int_{t_{j}}^{t_{j+1}}\left|\left(u_{k}^{j}\right)^{\Delta}(t)-u_{\Gamma_{j}}^{\Delta}(t)\right|^{2} \Delta t\right]^{1 / 2} \\
& \leq\left[\sum_{j=0}^{p}\left\|u_{k}^{j}-u^{j}\right\|_{H_{\Delta}^{1}\left(\left[t_{j}, t_{j+1}\right]_{\mathbb{T}}\right)}^{2}\right]^{1 / 2}
\end{aligned}
$$

we have $u_{k}$ converges to $u$ in $H$ as $k \rightarrow+\infty$. The proof is complete.

Lemma 3.3. If $\sum_{k=1}^{p}\left|A_{k}\right|<1$, then for any $u \in H$,

$$
\sum_{j=0}^{p} \int_{t_{j}}^{t_{j+1}}\left|u_{\Gamma_{j}}^{\sigma}(t)\right|^{2} \Delta t \leq R_{0}^{2} T\|u\|_{H}^{2}
$$

where $R_{0}$ is given in Lemma 3.1 .

Proof. For any $u \in H, t \in\left[t_{j}, t_{j+1}\right]_{\mathbb{T}}$, by Lemma 3.1, we have

$$
\left|u_{\Gamma_{j}}^{\sigma}(t)\right| \leq R_{0}\|u\|_{H}, \quad j=0,1, \ldots, p,
$$

which implies that

$$
\sum_{j=0}^{p} \int_{t_{j}}^{t_{j+1}}\left|u_{\Gamma_{j}}^{\sigma}(t)\right|^{2} \Delta t \leq R_{0}^{2} T\|u\|_{H}^{2}
$$

The proof is complete.

For any $u \in H$ satisfying (1.1)-(1.4), take $v \in H$ and multiply (1.1) by $v_{\Gamma_{j}}^{\sigma}$, then integrate it between $t_{j}$ and $t_{j+1}$ :

$$
-\int_{t_{j}}^{t_{j+1}} u^{\Delta \Delta}(t) v_{\Gamma_{j}}^{\sigma}(t) \Delta t=\int_{t_{j}}^{t_{j+1}} f\left(\sigma(t), u^{\sigma}(t)\right) v_{\Gamma_{j}}^{\sigma}(t) \Delta t .
$$


The first term is now

$$
\int_{t_{j}}^{t_{j+1}} u^{\Delta \Delta}(t) v_{\Gamma_{j}}^{\sigma}(t) \Delta t=u^{\Delta}\left(t_{j+1}^{-}\right) v_{\Gamma_{j}}\left(t_{j+1}^{-}\right)-u^{\Delta}\left(t_{j}^{+}\right) v_{\Gamma_{j}}\left(t_{j}^{+}\right)-\int_{t_{j}}^{t_{j+1}} u^{\Delta}(t) v_{\Gamma_{j}}^{\Delta}(t) \Delta t
$$

Hence, one gets

$$
\begin{aligned}
& -\sum_{j=0}^{p} \int_{t_{j}}^{t_{j+1}} u^{\Delta \Delta}(t) v_{\Gamma_{j}}^{\sigma}(t) \Delta t \\
& \quad=\sum_{j=0}^{p}\left[u^{\Delta}\left(t_{j}^{+}\right) v_{\Gamma_{j}}\left(t_{j}^{+}\right)-u^{\Delta}\left(t_{j+1}^{-}\right) v_{\Gamma_{j}}\left(t_{j+1}^{-}\right)\right]+\sum_{j=0}^{p} \int_{t_{j}}^{t_{j+1}} u^{\Delta}(t) v_{\Gamma_{j}}^{\Delta}(t) \Delta t \\
& \quad=\sum_{j=1}^{p}\left[u^{\Delta}\left(t_{j}^{+}\right) v\left(t_{j}^{+}\right)-u^{\Delta}\left(t_{j}^{-}\right) v\left(t_{j}^{-}\right)\right]+\sum_{j=0}^{p} \int_{t_{j}}^{t_{j+1}} u^{\Delta}(t) v_{\Gamma_{j}}^{\Delta}(t) \Delta t \\
& \quad=\sum_{j=1}^{p}\left[\left(1+A_{j}\right) u^{\Delta}\left(t_{j}^{+}\right)-u^{\Delta}\left(t_{j}^{-}\right)\right] v\left(t_{j}^{-}\right)+\sum_{j=0}^{p} \int_{t_{j}}^{t_{j+1}} u^{\Delta}(t) v_{\Gamma_{j}}^{\Delta}(t) \Delta t \\
& \quad=\sum_{j=1}^{p}\left(1+A_{j}\right) I_{j}\left(u\left(t_{j}^{-}\right)\right) v\left(t_{j}^{-}\right)+\sum_{j=0}^{p} \int_{t_{j}}^{t_{j+1}} u^{\Delta}(t) v_{\Gamma_{j}}^{\Delta}(t) \Delta t
\end{aligned}
$$

for all $u, v \in H$. Then we have

$$
\sum_{j=0}^{p} \int_{t_{j}}^{t_{j+1}} u^{\Delta}(t) v_{\Gamma_{j}}^{\Delta}(t) \Delta t-\sum_{j=0}^{p} \int_{t_{j}}^{t_{j+1}} f\left(\sigma(t), u^{\sigma}(t)\right) v_{\Gamma_{j}}^{\sigma}(t) \Delta t+\sum_{j=1}^{p}\left(1+A_{j}\right) I_{j}\left(u\left(t_{j}^{-}\right)\right) v\left(t_{j}^{-}\right)=0,
$$

for all $u, v \in H$.

This suggests that one defines $\varphi: H \rightarrow \mathbb{R}$, by

$$
\varphi(u)=\frac{1}{2} \sum_{j=0}^{p} \int_{t_{j}}^{t_{j+1}}\left|u_{\Gamma_{j}}^{\Delta}(t)\right|^{2} \Delta t-\sum_{j=0}^{p} \int_{t_{j}}^{t_{j+1}} F\left(\sigma(t), u_{\Gamma_{j}}^{\sigma}(t)\right) \Delta t+\sum_{j=1}^{p} \int_{0}^{u\left(t_{j}\right)} \bar{I}_{j}(s) d s,
$$

where $F(t, x)=\int_{0}^{x} f(t, s) d s$, and $\bar{I}_{j}=\left(1+A_{j}\right) I_{j}, \forall j=1,2, \ldots, p$. 
By a standard argument, one can prove that the functional $\varphi$ is continuously differentiable at any $u \in H$ and

$$
\left(\varphi^{\prime}(u), v\right)=\sum_{j=0}^{p} \int_{t_{j}}^{t_{j+1}} u_{\Gamma_{j}}^{\Delta}(t) v_{\Gamma_{j}}^{\Delta}(t) \Delta t-\sum_{j=0}^{p} \int_{t_{j}}^{t_{j+1}} f\left(\sigma(t), u_{\Gamma_{j}}^{\sigma}(t)\right) v_{\Gamma_{j}}^{\sigma}(t) \Delta t+\sum_{j=1}^{p} \bar{I}_{j}\left(u\left(t_{j}\right)\right) v\left(t_{j}\right),
$$

for all $u, v \in H$.

We call such critical points weak solutions of problem (1.1)-(1.4).

Let $E$ be a Banach space, $\varphi \in C^{1}(E, \mathbb{R})$, which means that $\varphi$ is a continuously Fréchetdifferentiable functional on E. $\varphi$ is said to satisfy the Palais-Smale condition (P-S condition) if any sequence $\left\{x_{n}\right\} \subset E$ such that $\left\{\varphi\left(x_{n}\right)\right\}$ is bounded and $\varphi^{\prime}\left(x_{n}\right) \rightarrow 0$ as $n \rightarrow \infty$, has a convergent subsequence in $E$.

Lemma 3.4 (Mountain pass theorem [26, Theorem 2.2], [27]). Let E be a real Hilbert space. Suppose $\varphi \in C^{1}(E, \mathbb{R})$, satisfies the P-S condition and the following assumptions:

$\left(l_{1}\right)$ there exist constants $\rho>0$ and $a>0$ such that $\varphi(x) \geq a$ for all $x \in \partial B_{\rho}$, where $B_{\rho}=\{x \in$ $\left.E \mid\|x\|_{E}<\rho\right\}$ which will be the open ball in $E$ with radius $\rho$ and centered at 0 ;

$\left(l_{2}\right) \varphi(0) \leq 0$ and there exists $x_{0} \notin B_{\rho}$ such that $\varphi\left(x_{0}\right) \leq 0$.

Then $\varphi$ possesses a critical value $c \geq a$. Moreover, $c$ can be characterized as

$$
c=\inf _{h \in \Gamma} \max _{s \in[0,1]} \varphi(h(s)),
$$

where

$$
\Gamma=\left\{h \in C([0,1] ; E) \mid h(0)=0, h(1)=x_{0}\right\}
$$

\section{Main Results}

Now we introduce some assumptions, which are used hereafter:

(H1) the function $f:[0, T]_{\mathbb{T}} \times \mathbb{R} \rightarrow \mathbb{R}$ is continuous;

(H2) $\lim _{x \rightarrow 0}(f(t, x) / x)=0$ holds uniformly for $t \in[0, T]_{\mathbb{T}}$;

(H3) there exist constants $\mu>2$ and $L>0$ such that

$$
0<\mu F(t, x) \leq x f(t, x), \quad \forall|x| \geq L
$$

(H4) there exist constants $\bar{M}_{j}$, with $0<\bar{M}<\min \left\{1 / 2 R_{0}^{2},(\mu-2) / R_{0}^{2}(\mu+2)\right\}$ such that

$$
\left|\left(1+A_{j}\right) I_{j}(x)\right| \leq \bar{M}_{j}|x|, \quad \forall x \in \mathbb{R}, j=1,2, \ldots, p,
$$

where $\bar{M}=\sum_{j=1}^{p} \bar{M}_{j}$, and $R_{0}=T^{1 / 2} /\left(1-\sum_{k=1}^{p}\left|A_{k}\right|\right)$. 
Remark 4.1. (H3) is the well-known Ambrosetti-Rabinowitz condition from the paper [27].

Lemma 4.2. Suppose that the conditions (H1)-(H4) are satisfied, then $\varphi$ satisfies the Palais-Smale condition.

Proof. Let $\left\{u_{k}\right\}$ be the sequence in $H$ satisfying that $\left\{\varphi\left(u_{k}\right)\right\}$ is bounded and $\varphi^{\prime}\left(u_{k}\right) \rightarrow 0$ as $k \rightarrow \infty$. Then there exists a constant $\beta>0$ such that

$$
\left|\varphi\left(u_{k}\right)\right| \leq \beta
$$

for every $k \in \mathbb{N}$. By $(H 3)$, we know that there exist constants $c_{1}>0, c_{2}>0$ such that

$$
F(t, x) \geq c_{1}|x|^{\mu}-c_{2}
$$

for all $x \in \mathbb{R}$. By (H4) and Lemma 3.1, we have

$$
\begin{aligned}
\sum_{j=1}^{p} \int_{0}^{u\left(t_{j}\right)} \bar{I}_{j}(s) d s & \geq-\sum_{j=1}^{p} \int_{\min \left\{0, u\left(t_{j}\right)\right\}}^{\max \left\{0, u\left(t_{j}\right)\right\}}\left|\bar{I}_{j}(s)\right| d s \\
& \geq-\frac{1}{2} \sum_{j=1}^{p} \bar{M}_{j}\left|u\left(t_{j}\right)\right|^{2} \\
& \geq-\frac{1}{2} \bar{M} R_{0}^{2}\|u\|_{H}^{2}, \\
\sum_{j=1}^{p} \bar{I}_{j}\left(u\left(t_{j}\right)\right) u\left(t_{j}\right) & \leq \sum_{j=1}^{p}\left|\bar{I}_{j}\left(u\left(t_{j}\right)\right)\right|\left|u\left(t_{j}\right)\right| \\
& \leq \sum_{j=1}^{p} \bar{M}_{j}\left|u\left(t_{j}\right)\right|^{2} \\
& \leq \bar{M} R_{0}^{2}\|u\|_{H}^{2},
\end{aligned}
$$

for all $u \in H$.

Set

$$
\begin{gathered}
\Omega_{k}^{j}=\left\{t \in\left[t_{j}, t_{j+1}\right]_{\mathbb{T}}|| u_{k}^{j}(\sigma(t)) \mid \geq L\right\}, \\
u_{k}^{j}(t):=\left(u_{k}\right)_{\Gamma_{j}}:= \begin{cases}u_{k}(t), & t \in\left(t_{j}, t_{j+1}\right]_{\mathbb{T}^{\prime}} \\
u_{k}\left(t_{j}^{+}\right), & t=t_{j},\end{cases}
\end{gathered}
$$

for $j=0,1, \ldots, p, k=1,2, \ldots$ 
It follows from (4.3)-(4.5), and (H3) that

$$
\begin{aligned}
\beta+\gamma\left\|u_{k}\right\|_{H} \geq & \varphi\left(u_{k}\right)-\frac{1}{\mu}\left(\varphi^{\prime}\left(u_{k}\right), u_{k}\right) \\
= & \left(\frac{1}{2}-\frac{1}{\mu}\right)\left\|u_{k}\right\|_{H}^{2}+\sum_{j=1}^{p} \int_{0}^{u_{k}\left(t_{j}\right)} \bar{I}_{j}(s) d s-\frac{1}{\mu} \sum_{j=1}^{p} \bar{I}_{j}\left(u_{k}\left(t_{j}\right)\right) u_{k}\left(t_{j}\right) \\
& -\sum_{j=0}^{p} \int_{t_{j}}^{t_{j+1}}\left[F\left(\sigma(t), u_{k}^{j}(\sigma(t))\right)-f\left(\sigma(t), u_{k}^{j}(\sigma(t))\right) u_{k}^{j}(\sigma(t))\right] \Delta t \\
= & \left(\frac{1}{2}-\frac{1}{\mu}\right)\left\|u_{k}\right\|_{H}^{2}+\sum_{j=1}^{p} \int_{0}^{u_{k}\left(t_{j}\right)} \bar{I}_{j}(s) d s-\frac{1}{\mu} \sum_{j=1}^{p} \bar{I}_{j}\left(u_{k}\left(t_{j}\right)\right) u_{k}\left(t_{j}\right) \\
& -\sum_{j=0}^{p} \int_{\Omega_{k}^{j}}\left[F\left(\sigma(t), u_{k}^{j}(\sigma(t))\right)-f\left(\sigma(t), u_{k}^{j}(\sigma(t))\right) u_{k}^{j}(\sigma(t))\right] \Delta t \\
& -\sum_{j=0}^{p} \int_{\left[t_{j}, t_{j+1}\right]_{\mathbb{T}} \backslash \Omega_{k}^{j}}\left[F\left(\sigma(t), u_{k}^{j}(\sigma(t))\right)-f\left(\sigma(t), u_{k}^{j}(\sigma(t))\right) u_{k}^{j}(\sigma(t))\right] \Delta t \\
\geq & \left(\frac{1}{2}-\frac{1}{\mu}-\frac{\bar{M} R_{0}^{2}}{2}-\frac{\bar{M} R_{0}^{2}}{\mu}\right)\|u\|_{H}^{2}-c_{3},
\end{aligned}
$$

for some constants $\gamma>0, c_{3}>0$, which implies that $\left\{\left\|u_{k}\right\|_{H}\right\}$ is bounded by the fact that $\mu>2, \bar{M}<(\mu-2) / R_{0}^{2}(\mu+2)$.

Then $\left\{\left\|u_{k}^{j}\right\|_{H_{\Delta}^{1}\left(\left[t_{j}, t_{j+1}\right]_{\mathbb{T}}\right)}\right\}$ is bounded in $H_{\Delta}^{1}\left(\left[t_{j}, t_{j+1}\right]_{\mathbb{T}}\right)$ for $j=0,1, \ldots, p$. Therefore, there exists a subsequence $\left\{u_{k}\right\}$ (for simplicity denoted again by $\left\{u_{k}\right\}$ ) such that $\left\{u_{k}^{j}\right\}$ converges weakly to $u^{j}$ in $H_{\Delta}^{1}\left(\left[t_{j}, t_{j+1}\right]_{\mathbb{T}}\right)$, and by Lemma 2.6, $\left\{u_{k}^{j}\right\}$ converges strongly to $u^{j}$ in $C\left(\left[t_{j}, t_{j+1}\right]_{\mathbb{T}}\right)$, that is, $\left\|u_{k}^{j}-u^{j}\right\|_{C\left(\left[t_{j}, t_{j+1}\right]_{\mathbb{T}}\right)} \rightarrow 0$ as $k \rightarrow+\infty$ for all $j=0,1, \ldots, p$.

Set

$$
u(t):= \begin{cases}u^{j}(t), & t \in\left(t_{j}, t_{j+1}\right]_{\mathbb{T}^{\prime}} j=0,1, \ldots, p, \\ u^{j-1}\left(t_{j}\right), & t=t_{j}, j=0,1, \ldots, p .\end{cases}
$$

In a similar way to Lemma 3.2 , one can prove that $u \in H, u_{\Gamma_{j}}=u^{j}, j=0,1, \ldots, p$. 
For any $v \in H$, we have

$$
\begin{aligned}
\left(v, u_{k}\right)_{H} & =\sum_{j=0}^{p} \int_{t_{j}}^{t_{j+1}} v_{\Gamma_{j}}^{\Delta}(t)\left(u_{k}^{j}\right)^{\Delta}(t) \Delta t \\
& =\sum_{j=0}^{p}\left[\left(v_{\Gamma_{j}}, u_{k}^{j}\right)_{H\left(\left[t_{j}, t_{j+1}\right]_{\mathbb{T}}\right)}-\int_{t_{j}}^{t_{j+1}} v_{\Gamma_{j}}(t) u_{k}^{j}(t) \Delta t\right] \\
& \longrightarrow \sum_{j=0}^{p}\left[\left(v_{\Gamma_{j}}, u^{j}\right)_{H\left(\left[t_{j}, t_{j+1}\right]_{\mathbb{T}}\right)}-\int_{t_{j}}^{t_{j+1}} v_{\Gamma_{j}}(t) u^{j}(t) \Delta t\right] \\
& =\sum_{j=0}^{p} \int_{t_{j}}^{t_{j+1}} v_{\Gamma_{j}}^{\Delta}(t)\left(u^{j}\right)^{\Delta}(t) \Delta t=(v, u)_{H^{\prime}}
\end{aligned}
$$

which implies that $\left\{u_{k}\right\}$ converges weakly to $u$ in $H$.

By (3.22) and (3.23), we have

$$
\begin{aligned}
\left(\varphi^{\prime}\left(u_{k}\right)\right. & \left.-\varphi^{\prime}(u), u_{k}-u\right) \\
= & \sum_{j=0}^{p} \int_{t_{j}}^{t_{j+1}}\left[\left(u_{k}^{j}\right)^{\Delta}(t)-u_{\Gamma_{j}}^{\Delta}(t)\right]^{2} \Delta t \\
& -\sum_{j=0}^{p} \int_{t_{j}}^{t_{j+1}}\left[f\left(\sigma(t), u_{k}^{j}(\sigma(t))\right)-f\left(\sigma(t), u_{\Gamma_{j}}^{\sigma}(t)\right)\right]\left[u_{k}^{j}(\sigma(t))-u_{\Gamma_{j}}^{\sigma}(t)\right] \Delta t \\
& +\sum_{j=1}^{p}\left[\bar{I}_{j}\left(u_{k}\left(t_{j}\right)\right)-\bar{I}_{j}\left(u\left(t_{j}\right)\right)\right]\left[u_{k}\left(t_{j}\right)-u\left(t_{j}\right)\right] \\
= & \sum_{j=0}^{p} \int_{t_{j}}^{t_{j+1}}\left[\left(u_{k}^{j}\right)^{\Delta}(t)-u_{\Gamma_{j}}^{\Delta}(t)\right]^{2} \Delta t \\
& -\sum_{j=0}^{p} \int_{t_{j}}^{t_{j+1}}\left[f\left(\sigma(t), u_{k}^{j}(\sigma(t))\right)-f\left(\sigma(t), u^{j}(\sigma(t))\right)\right]\left[u_{k}^{j}(\sigma(t))-u^{j}(\sigma(t))\right] \Delta t \\
& +\sum_{j=1}^{p}\left[\bar{I}_{j}\left(u_{k}^{j-1}\left(t_{j}\right)\right)-\bar{I}_{j}\left(u^{j-1}\left(t_{j}\right)\right)\right]\left[u_{k}^{j-1}\left(t_{j}\right)-u^{j-1}\left(t_{j}\right)\right] .
\end{aligned}
$$

By the fact that $\left(\varphi^{\prime}\left(u_{k}\right)-\varphi^{\prime}(u), u_{k}-u\right) \rightarrow 0$ as $k \rightarrow \infty$, and the continuity of $f$ and $\bar{I}_{j}$, on $[0, T]_{\mathbb{T}}, j=1,2, \ldots, p$, we conclude

$$
\sum_{j=0}^{p} \int_{t_{j}}^{t_{j+1}}\left[\left(u_{k}^{j}\right)^{\Delta}(t)-u_{\Gamma_{j}}^{\Delta}(t)\right]^{2} \Delta t \longrightarrow 0 \quad \text { as } k \longrightarrow \infty,
$$


that is,

$$
\left\|u_{k}-u\right\|_{H} \longrightarrow 0 \quad \text { as } k \longrightarrow \infty
$$

Thus, $\left\{u_{k}\right\}$ possesses a convergent subsequence in $H$. Then, the P-S condition is now satisfied.

Theorem 4.3. Suppose that (H1)-(H4) hold. Then problem (1.1)-(1.4) has at least one nontrivial weak solution on $H$.

Proof. In order to show that $\varphi$ has at least one nonzero critical point, it suffices to check the conditions $\left(l_{1}\right)$ and $\left(l_{2}\right)$. It follows from $(H 2)$ that there is a constant $\delta>0$ such that

$$
|f(t, s)| \leq \frac{1}{2 R_{0}^{2} T}|s|
$$

for all $0<|s| \leq \delta$ and $t \in[0, T]_{\mathbb{T}}$. Hence, we have

$$
\begin{aligned}
F\left(\sigma(t), u^{\sigma}(t)\right) & =\int_{0}^{u^{\sigma}(t)} f(\sigma(t), s) d s \\
& \leq \int_{\min \left\{0, u^{\sigma}(t)\right\}}^{\max \left\{0, u^{\sigma}(t)\right\}}|f(\sigma(t), s)| d s \\
& \leq \frac{1}{2 R_{0}^{2} T} \int_{\min \left\{0, u^{\sigma}(t)\right\}}^{\max \left\{0, u^{\sigma}(t)\right\}}|s| d s \\
& \leq \frac{1}{4 R_{0}^{2} T}\left|u^{\sigma}(t)\right|^{2},
\end{aligned}
$$

for all $\sup _{t \in[0, T]_{\mathbb{T}}}|u(t)| \leq \delta$ and $t \in[0, T]_{\mathbb{T}}$. By Lemma 3.3, we obtain

$$
\begin{aligned}
\sum_{j=0}^{p} \int_{t_{j}}^{t_{j+1}} F\left(\sigma(t), u_{\Gamma_{j}}^{\sigma}(t)\right) \Delta t & \leq \frac{1}{4 R_{0}^{2} T} \sum_{j=0}^{p} \int_{t_{j}}^{t_{j+1}}\left|u_{\Gamma_{j}}^{\sigma}(t)\right|^{2} \Delta t \\
& \leq \frac{1}{4}\|u\|_{H^{\prime}}^{2}
\end{aligned}
$$

for all $\sup _{t \in[0, T]_{\mathbb{T}}}|u(t)| \leq \delta$ and $t \in[0, T]_{\mathbb{T}}$. It follows from (4.5) and (4.16) that

$$
\begin{aligned}
\varphi(u) & \geq \frac{1}{2}\|u\|_{H}^{2}-\frac{1}{4}\|u\|_{H}^{2}-\frac{1}{2} \bar{M} R_{0}^{2}\|u\|_{H}^{2} \\
& =\frac{1}{2}\left(\frac{1}{2}-\bar{M} R_{0}^{2}\right)\|u\|_{H^{\prime}}^{2}
\end{aligned}
$$


for all $\sup _{t \in[0, T]_{\mathbb{T}}}|u(t)| \leq \delta$ and $t \in[0, T]_{\mathbb{T}}$. Therefore, by (3.6), one gets

$$
\varphi(u) \geq \alpha,
$$

for all $u \in \partial B_{\rho}$, where $B_{\rho}=\left\{u \in H \mid\|u\|_{H}<\rho=\delta / R_{0}\right\}$ and $\alpha=\left(\delta^{2} / 2 R_{0}^{2}\right)\left((1 / 2)-\bar{M} R_{0}^{2}\right)>0$. Then $\left(l_{1}\right)$ is verified. Next we verify $\left(l_{2}\right)$. By $(4.4)$, one has

$$
\sum_{j=0}^{p} \int_{t_{j}}^{t_{j+1}} F\left(\sigma(t), u_{\Gamma_{j}}^{\sigma}(t)\right) \Delta t \geq c_{1} \sum_{j=0}^{p} \int_{t_{j}}^{t_{j+1}}\left|u_{\Gamma_{j}}^{\sigma}(t)\right|^{\mu} \Delta t-c_{2} T,
$$

for all $u \in H$, and by (H4) and Lemma 3.1, we have

$$
\begin{aligned}
\sum_{j=1}^{p} \int_{0}^{u\left(t_{j}\right)} \bar{I}_{j}(s) d s & \leq \sum_{j=1}^{p} \int_{\min 0, u\left(t_{j}\right)}^{\max 0, u\left(t_{j}\right)}\left|\bar{I}_{j}(s)\right| d s \\
& \leq \frac{1}{2} \sum_{j=1}^{p} \bar{M}_{j}\left|u\left(t_{j}\right)\right|^{2} \\
& \leq c_{4}\|u\|_{H^{\prime}}^{2}
\end{aligned}
$$

for all $u \in H$ and some positive constant $c_{4}$.

Let $v_{0} \in H$ and $\left\|v_{0}\right\|_{H}=1$. For any $\tau>0$, by (4.19) and (4.20), one obtains

$$
\begin{aligned}
\varphi\left(\tau v_{0}\right) & =\frac{\tau^{2}}{2}\left\|v_{0}\right\|_{H}^{2}-\sum_{j=0}^{p} \int_{t_{j}}^{t_{j+1}} F\left(\sigma(t), \tau\left(v_{0}\right)_{\Gamma_{j}}^{\sigma}(t)\right) \Delta t+\sum_{j=1}^{p} \int_{0}^{\tau v_{0}\left(t_{j}\right)} \bar{I}_{j}(s) d s \\
& \leq \frac{\tau^{2}}{2}\left\|v_{0}\right\|_{H}^{2}-c_{1} \tau^{\mu} \sum_{j=0}^{p} \int_{t_{j}}^{t_{j+1}}\left|\left(v_{0}\right)_{\Gamma_{j}}^{\sigma}(t)\right|^{\mu} \Delta t+c_{2} T+c_{4} \tau^{2}\left\|v_{0}\right\|_{H}^{2} \\
& \leq\left(\frac{1}{2}+c_{4}\right) \tau^{2}\left\|v_{0}\right\|_{H}^{2}-c_{1} \tau^{\mu} \sum_{j=0}^{p} \int_{t_{j}}^{t_{j+1}}\left|\left(v_{0}\right)_{\Gamma_{j}}^{\sigma}(t)\right|^{\mu} \Delta t+c_{2} T,
\end{aligned}
$$

which implies that

$$
\varphi\left(\tau v_{0}\right) \longrightarrow-\infty,
$$

as $\tau \rightarrow \infty$ for $\mu>2$. Hence, we can choose sufficiently large $\tau_{0}>\rho$ such that $u_{0}=\tau_{0} v_{0} \in H$, $u_{0} \notin B_{\rho}$, and $\varphi\left(u_{0}\right)<0$. Assumption $\left(l_{2}\right)$ is verified. Theorem 4.3 is now proved. 
Example 4.4. Let $[0, T]_{\mathbb{T}}=[0,0.01] \cup[0.02,0.05], t_{1}=0.02, T=0.05$. Then the system

$$
\begin{gathered}
-u^{\Delta \Delta}(t)=4[u(\sigma(t))]^{3} \exp [u(\sigma(t))]^{4}, \quad t \in[0, T]_{\mathbb{T}}, t \neq t_{1}, \\
u\left(t_{1}^{+}\right)-u\left(t_{1}^{-}\right)=\frac{1}{2} u\left(t_{1}^{-}\right) \\
u^{\Delta}\left(t_{1}^{+}\right)-u^{\Delta}\left(t_{1}^{-}\right)=u\left(t_{1}^{-}\right)-\frac{1}{3} u^{\Delta}\left(t_{1}^{-}\right) \\
u(0)=u(T)=0
\end{gathered}
$$

is solvable.

\section{Acknowledgment}

This research is supported by the National Natural Science Foundation of China (no. 10561004).

\section{References}

[1] S. Hilger, "Analysis on measure chains-a unified approach to continuous and discrete calculus," Results in Mathematics, vol. 18, no. 1-2, pp. 18-56, 1990.

[2] R. P. Agarwal, M. Bohner, and P. J. Y. Wong, "Sturm-Liouville eigenvalue problems on time scales," Applied Mathematics and Computation, vol. 99, no. 2-3, pp. 153-166, 1999.

[3] R. Agarwal, M. Bohner, D. O’Regan, and A. Peterson, "Dynamic equations on time scales: a survey," Journal of Computational and Applied Mathematics, vol. 141, no. 1-2, pp. 1-26, 2002.

[4] P. Amster and P. D. Nápoli, "Variational methods for two resonant problems on time scales," International Journal of Difference Equations, vol. 2, no. 1, pp. 1-12, 2007.

[5] D. R. Anderson, "Eigenvalue intervals for a two-point boundary value problem on a measure chain," Journal of Computational and Applied Mathematics, vol. 141, no. 1-2, pp. 57-64, 2002.

[6] D. R. Anderson and R. I. Avery, "An even-order three-point boundary value problem on time scales," Journal of Mathematical Analysis and Applications, vol. 291, no. 2, pp. 514-525, 2004.

[7] F. M. Atici and G. Sh. Guseinov, "On Green's functions and positive solutions for boundary value problems on time scales," Journal of Computational and Applied Mathematics, vol. 141, no. 1-2, pp. 75-99, 2002.

[8] A. Cabada, "Extremal solutions and Green's functions of higher order periodic boundary value problems in time scales," Journal of Mathematical Analysis and Applications, vol. 290, no. 1, pp. 35-54, 2004.

[9] Z. He, "Existence of two solutions of $m$-point boundary value problem for second order dynamic equations on time scales," Journal of Mathematical Analysis and Applications, vol. 296, no. 1, pp. 97-109, 2004.

[10] L. Jiang and Z. Zhou, "Existence of weak solutions of two-point boundary value problems for secondorder dynamic equations on time scales," Nonlinear Analysis: Theory, Methods \& Applications, vol. 69, no. 4, pp. 1376-1388, 2008.

[11] S. G. Topal, "Second-order periodic boundary value problems on time scales," Computers $\mathcal{E}$ Mathematics with Applications, vol. 48, no. 3-4, pp. 637-648, 2004.

[12] A. Cabada, "Existence results for $\varphi$-Laplacian boundary value problems on time scales," Advances in Difference Equations, vol. 2006, Article ID 21819, 11 pages, 2006.

[13] F. A. Davidson and B. P. Rynne, "The formulation of second-order boundary value problems on time scales," Advances in Difference Equations, vol. 2006, Article ID 31430, 10 pages, 2006.

[14] B. Karna and B. A. Lawrence, "An existence result for a multipoint boundary value problem on a time scale," Advances in Difference Equations, vol. 2006, Article ID 63208, 8 pages, 2006. 
[15] R. P. Agarwal, V. Otero-Espinar, K. Perera, and D. R. Vivero, "Multiple positive solutions in the sense of distributions of singular BVPs on time scales and an application to Emden-Fowler equations," Advances in Difference Equations, vol. 2008, Article ID 796851, 13 pages, 2008.

[16] M. Benchohra, J. Henderson, and S. K. Ntouyas, "Eigenvalue problems for systems of nonlinear boundary value problems on time scales," Advances in Difference Equations, vol. 2007, Article ID 031640, 10 pages, 2007.

[17] V. Lakshmikantham, D. D. Baĭnov, and P. S. Simeonov, Theory of Impulsive Differential Equations, vol. 6 of Series in Modern Applied Mathematics, World Scientific, Teaneck, NJ, USA, 1989.

[18] A. M. Samořlenko and N. A. Perestyuk, Impulsive Differential Equations, vol. 14 of World Scientific Series on Nonlinear Science. Series A: Monographs and Treatises, World Scientific, River Edge, NJ, USA, 1995.

[19] M. Benchohra, S. K. Ntouyas, and A. Ouahab, “Existence results for second order boundary value problem of impulsive dynamic equations on time scales," Journal of Mathematical Analysis and Applications, vol. 296, no. 1, pp. 65-73, 2004.

[20] J. J. Nieto and D. O'Regan, "Variational approach to impulsive differential equations," Nonlinear Analysis: Real World Applications, vol. 10, no. 2, pp. 680-690, 2009.

[21] H. Zhang and Z. X. Li, "Periodic solutions of second-order nonautonomous impulsive differential equations," International Journal of Qualitative Theory of Differential Equations and Applications, vol. 2, no. 1, pp. 112-124, 2008.

[22] M. Bohner and A. Peterson, Dynamic Equations on Time Scales: An Introdution with Applications, Birkhäuser, Boston, Mass, USA, 2001.

[23] M. Bohner and A. Peterson, Dynamic Equations on Time Scales, Birkhäuser, Boston, Mass, USA, 2001.

[24] R. P. Agarwal, V. Otero-Espinar, K. Perera, and D. R. Vivero, “Basic properties of Sobolev's spaces on time scales," Advances in Difference Equations, vol. 2006, Article ID 38121, 14 pages, 2006.

[25] R. Agarwal, M. Bohner, and A. Peterson, "Inequalities on time scales: a survey," Mathematical Inequalities $\mathcal{E}$ Applications, vol. 4, no. 4, pp. 535-557, 2001.

[26] P. H. Rabinowitz, Minimax Methods in Critical Point Theory with Applications to Differential Equations, vol. 65 of CBMS Regional Conference Series in Mathematics, CBMS AMS, Providence, RI, USA, 1986.

[27] A. Ambrosetti and P. H. Rabinowitz, "Dual variational methods in critical point theory and applications," Journal of Functional Analysis, vol. 14, no. 4, pp. 349-381, 1973. 